\title{
Linked Data Tools for Managing Taxonomic Databases
}

\author{
Johan Liljeblad ${ }^{\ddagger}$, Tapani Lahti§ ${ }^{\S}$ Matts Djos। \\ ‡ Swedish Species Information Centre, Uppsala, Sweden \\ $\S$ University of Helsinki, Helsinki, Finland \\ | ArtDatabanken, Uppsala, Sweden
}

Corresponding author: Johan Liljeblad (cynips@gmail.com)

Received: 14 Jun 2019 | Published: 21 Jun 2019

Citation: Liljeblad J, Lahti T, Djos M (2019) Linked Data Tools for Managing Taxonomic Databases. Biodiversity

Information Science and Standards 3: e37329. https://doi.org/10.3897/biss.3.37329

\begin{abstract}
Taxonomic information is dynamic, i.e. changes are made continuously, so scientific names are insufficient to track changes in taxon circumscription. The principles of Linked Open Data (LOD), as defined by the World Wide Web Consortium, can be applied for documenting the relationships of taxon circumscriptions over time and between checklists of taxa.

In our scheme, each checklist and each taxon in the checklist is assigned a globally unique, persistent identifier. According to the LOD principles, HTTP Uniform Resource Identifiers (URIs) are used as identifiers, providing both human-readable (HTML) and machine-readable $(\mathrm{XML})$ responses for client requests.

Common vocabularies are needed in machine-readable responses to HTTP URIs. We use SKOS (Simple Knowledge Organization System) as a basic vocabulary for describing checklists as instances of class skos:ConceptScheme, and taxa as instances of class skos:Concept. Set relationships between taxon circumscriptions are described using the properties skos:broader and skos:narrower. Darwin Core vocabulary is used for describing taxon properties, such as scientific names, taxonomic ranks and authorship string, in the checklists.
\end{abstract}


Instead of directly linking taxon circumscriptions between checklists, we define a HTTP URI for each unique circumscription. This common identifier is then mapped to internal checklist identifiers matching the circumscription using the property skos:exactMatch. For the management of the URIs, the domain name TAXONID.ORG has been registered.

In a pilot study, our approach has been applied to linking taxon circumscriptions of selected taxa between the national checklists of Sweden and Finland. In the future, national checklists from other Nordic/Baltic countries (Norway, Denmark, Iceland, Estonia) can be easily linked together as well. The work is part of the NeIC DeepDive project (neic.no).

\section{Keywords}

checklist, identifier, taxon circumscription, matching, linking

\section{Presenting author}

Johan Liljeblad

\section{Presented at}

Biodiversity_Next 2019 